\title{
Overview of the prevalence, impact, and management of depression and anxiety in chronic obstructive pulmonary disease
}

This article was published in the following Dove Press journal:

International Journal of COPD

13 November 2014

Number of times this article has been viewed

\section{Maria Panagioti' \\ Charlotte Scott' \\ Amy Blakemore ${ }^{1,2}$ \\ Peter A Coventry ${ }^{3}$}

'National Institute for Health Research School for Primary Care Research, Centre for Primary Care, Institute of Population Health, Manchester Academic Health Science Centre, University of Manchester, ${ }^{2}$ Department of Psychiatry, Manchester Mental Health and Social Care Trust, Manchester Royal Infirmary, ${ }^{3}$ National Institute for Health Research Collaboration for Leadership in Applied Health Research and Care - Greater Manchester and Manchester Academic Health Science Centre, University of Manchester, Manchester, UK
Correspondence: Peter A Coventry National Institute for Health Research Collaboration for Leadership in Applied Health Research and Care, Manchester Academic Health Science Centre, University of Manchester, Manchester MI3 9PL, UK

Tel +44 016 I306 7653

Fax +44 $016 \quad 12757600$

Email peter.a.coventry@manchester.ac.uk
Abstract: More than one third of individuals with chronic obstructive pulmonary disease (COPD) experience comorbid symptoms of depression and anxiety. This review aims to provide an overview of the burden of depression and anxiety in those with COPD and to outline the contemporary advances and challenges in the management of depression and anxiety in COPD. Symptoms of depression and anxiety in COPD lead to worse health outcomes, including impaired health-related quality of life and increased mortality risk. Depression and anxiety also increase health care utilization rates and costs. Although the quality of the data varies considerably, the cumulative evidence shows that complex interventions consisting of pulmonary rehabilitation interventions with or without psychological components improve symptoms of depression and anxiety in COPD. Cognitive behavioral therapy is also an effective intervention for managing depression in COPD, but treatment effects are small. Cognitive behavioral therapy could potentially lead to greater benefits in depression and anxiety in people with COPD if embedded in multidisciplinary collaborative care frameworks, but this hypothesis has not yet been empirically assessed. Mindfulness-based treatments are an alternative option for the management of depression and anxiety in people with long-term conditions, but their efficacy is unproven in COPD. Beyond pulmonary rehabilitation, the evidence about optimal approaches for managing depression and anxiety in COPD remains unclear and largely speculative. Future research to evaluate the effectiveness of novel and integrated care approaches for the management of depression and anxiety in COPD is warranted.

Keywords: chronic obstructive pulmonary disease, depression and anxiety, health outcomes, pulmonary rehabilitation, cognitive behavioral therapy, multidisciplinary case management

\section{Introduction}

\section{Prevalence and symptoms of depression and anxiety}

Depression is a common mental health problem accompanied by a high degree of emotional distress and functional impairment. ${ }^{1}$ The two main symptoms of major depression include depressed mood and loss of interest or pleasure in daily activities. Additional symptoms of depression include fatigue or loss of energy, significant changes in weight, appetite and sleep, guilt/worthlessness, lack of concentration, pessimism about the future, and suicidality. According to the Fifth Edition of the Statistical Manual of Mental Disorders, a diagnosis of major depression is assigned if at least one of two main symptoms and five symptoms in total are present for at least 2 weeks and cause clinically significant impairment in social, occupational, or other important areas of functioning. ${ }^{2,3}$ Major depressive disorder accounted for $8.2 \%$ of years living with disability in 2010, making it the second leading direct cause of global disease burden. ${ }^{4}$ 
Anxiety is also a common mental health problem and is associated with physical and psychological discomfort. All the anxiety disorders share common symptoms, such as fear, anxiety, and avoidance. Other anxiety-related symptoms include fatigue, restlessness, irritability, sleep disturbances, reduced concentration and memory, and muscle tension. ${ }^{3}$ Among the anxiety disorders, the most common are specific or social phobias and generalized anxiety disorder. ${ }^{5}$

Depression and anxiety often co-occur; it is estimated that at least half of people with depression also have anxiety. In fact, there is evidence that a mixed state of depression and anxiety is more prevalent than depression alone. ${ }^{6}$ The prevalence of depression and anxiety is two to three times higher in people with chronic (long-term) medical conditions. ${ }^{7}$ People with a long-term condition and depression/ anxiety have worse health status than people with depression/ anxiety alone, or people with any combination of long-term conditions without depression. ${ }^{8}$

\section{Prevalence of depression and anxiety in COPD}

A recent meta-analysis that included 39,587 individuals with COPD and 39,431 controls found that one in four COPD patients experienced clinically significant depressive symptoms compared with less than one in eight of the controls (24.6\%, 95\% confidence interval [CI] 20.0-28.6 versus $11.7 \%, 95 \%$ CI 9.0-15.1). ${ }^{9}$ These estimates are consistent with the findings of previous qualitative and quantitative reviews that assessed the prevalence of depressive symptoms in COPD. ${ }^{10-12}$ Clinical anxiety has also been recognized as a significant problem in COPD, with an estimated prevalence of up to $40 \%{ }^{12,13}$ Additionally, COPD patients are ten times more likely to experience panic disorder or panic attacks compared with general population samples. ${ }^{14}$ Of note, the great variability of methods used to assess depression and anxiety in the literature makes it difficult to reach a consensus about the prevalence of depression and anxiety in COPD. Future research should quantify whether prevalence rates for depression and anxiety in COPD are significantly different among samples identified by self-rated or standardized interview methods.

The causes of depression and anxiety in COPD are likely to be multifactorial, but importantly disease severity does not appear to affect the levels of anxiety and depression in COPD patients. ${ }^{15}$ Rather, subjective ratings of health-related quality of life (HRQoL), dyspnea, and reduced exercise capacity potentially underlie the development of symptoms of depression and anxiety in COPD. ${ }^{16,17}$ Additionally, depression and anxiety are more often reported in women than in men with COPD, but differences in perceived symptom control and severity of dyspnea symptoms appear to account for this finding. ${ }^{18,19}$ The meta-analysis by Zhang et al showed no differences in the prevalence of depression in COPD between studies of Western and non-Western populations. ${ }^{9}$ However, there is evidence that certain subgroups of British South Asians have higher rates of depression, but it is not clear what contribution somatic, genetic, or lifestyle factors play in accounting for health differentials between different ethnic groups. ${ }^{20-22}$ Further research is needed to examine the effects of ethnicity and nationality on the prevalence rates of depression and anxiety in COPD.

\section{Impact of depression and anxiety on health-related quality of life}

HRQoL is a multifaceted concept that is uniquely linked to health or illness, and includes a number of distinct domains corresponding to the physical, social, and psychological impact of illness. ${ }^{23}$ A considerable number of published empirical studies and systematic reviews offer robust evidence that symptoms of depression and anxiety are associated with poorer HRQoL in COPD. ${ }^{24-26}$ However, this evidence is mainly derived from cross-sectional studies, which preclude any temporal or causal inferences being made about the association between HRQoL and depression and anxiety in COPD. A recent systematic review by Blakemore et al has examined the longitudinal impact of depression and anxiety on HRQoL. This review found that both depression and anxiety at baseline are significantly associated with worsening levels of HRQoL at 1 year follow-up (pooled $r=0.48,95 \%$ CI 0.37-0.57, $P<0.001$; pooled $r=0.36,95 \%$ CI $0.23-0.48$, $P<0.001$; for depression and anxiety, respectively). ${ }^{27}$ The findings of this review suggest that HRQoL may be a worthwhile target for interventions aiming to improve the psychological health of people with COPD. ${ }^{27}$

\section{Impact of depression and anxiety on health care utilization}

Comorbid depression and anxiety in COPD is associated with a disproportionate increase in health care utilization rates and costs. A population-based study among people with six chronic conditions (including COPD) showed that comorbid depression doubled the likelihood of health care utilization, functional disability, and work absence. ${ }^{28}$ Similarly, a US study among a managed care population showed that COPD patients with comorbid depression were $77 \%$ more likely 
to have a COPD-related hospitalization, $48 \%$ more likely to have an emergency room visit, and $60 \%$ more likely to have a hospitalization/emergency room visit compared with COPD patients without comorbid depression. ${ }^{29}$ Other studies in this area suggest that depression in COPD leads to excessive health care utilization rates and costs, including longer hospital stay after acute exacerbation, ${ }^{30}$ increased risk of exacerbation and hospital admission, ${ }^{31,32}$ and hospital readmission. ${ }^{33}$ Comorbid anxiety and panic disorder in COPD is also associated with increased risk of exacerbations, relapse within 1 month of receiving emergency treatment, ${ }^{34}$ and hospital readmission. ${ }^{35}$

Evidence from systematic reviews and empirical studies suggests that the presence of mental health problems (including depression and anxiety) inflates the costs of care for long-term conditions by at least $45 \%$ after controlling for severity of physical illness. ${ }^{36-41}$ In COPD in particular, a recent study showed that comorbid depression and anxiety significantly inflated average annual all-cause health care costs $(\$ 23,759$ versus $\$ 17,765$ per patient, $P<0.001)$ and COPD total health care costs $(\$ 3,185$ versus $\$ 2,680$ per patient; $P<0.001) .{ }^{29}$ Moreover, Howard et al found that the addition of a psychological component in a breathlessness clinic for COPD led to savings of $£ 837$ per patient 6 months after the intervention (which were mainly attributed to lower emergency room visits and fewer hospital bed days). ${ }^{42}$

\section{Impact of depression and anxiety on mortality in COPD}

COPD is the fourth leading cause of morbidity worldwide and is expected to be the third leading cause of mortality by $2020 .^{43}$ The bulk of studies exploring mortality in patients with COPD have mainly focused on physiologic prognostic factors. ${ }^{44}$ In the past decade, an increasing number of prognostic studies have indicated that mental health problems also contribute significantly to mortality risk in COPD. Depression is a particularly strong predictor for mortality in COPD (odds ratios ranging from 1.9 to 2.7$)^{30,45,46}$ and its predictive ability persists over and above the effects of other prognostic factors, including physiological factors, demographic factors, and disease severity. ${ }^{47,48}$ Moreover, preliminary evidence suggests that depression and anxiety interact with other risk factors (eg, physiological factors and smoking) to produce stronger combined effects on mortality risk in COPD. ${ }^{49}$ On these grounds, the risk for death in COPD might be better ascertained by the simultaneous consideration of physiological and psychological prognostic factors and the awareness that the impact of these factors on mortality could be cumulative.

\section{Managing depression and anxiety in COPD}

There is a growing consensus in respiratory medicine that the therapeutic focus in COPD should move beyond disease modification and survival alone, and include assessment and improvement of patient-centered outcomes, including health status and psychological health. ${ }^{50,51}$ Likewise, in recognition of the increased health and economic burden associated with aging populations with long-term conditions, governments and policymakers are equally keen to promote approaches that integrate physical and mental health care, leading to improved patient outcomes, reduced unscheduled care, and reduced health care costs. ${ }^{52}$ In the UK, for example, the National Institute for Health and Care Excellence has published clinical guidelines that recommend the use of stepped approaches to psychological and/or pharmacological treatment of depression in adults in primary care $;^{53}$ similar guidelines have been published to underpin comparable approaches for managing depression in people with long-term conditions. ${ }^{54}$ Treatments include psychological therapies based on a cognitive and behavioral framework with or without antidepressant medication. ${ }^{55}$ But while there is good evidence that psychological therapies are as effective as antidepressants, ${ }^{56}$ and that patients prefer psychological therapies, ${ }^{57}$ treatment of depression and anxiety in people with long-term conditions is not as optimal as it could be. This is especially true in primary care where the majority of COPD patients are managed. Time-limited consultations that prioritize physical health mean that depression and anxiety remain underdetected and undertreated in people with COPD. ${ }^{58}$

Outside of general practice-led primary care, the most promising intervention to meet the challenges of managing depression in people with COPD is pulmonary rehabilitation. There is growing evidence that pulmonary rehabilitation can not only improve HRQoL and exercise capacity, ${ }^{59,60}$ but depression and anxiety too. ${ }^{61}$ The next section of this overview offers a detailed summary of the comparative effectiveness of pulmonary rehabilitation and other nonpharmacological interventions for managing depression in people with COPD.

\section{Multidisciplinary pulmonary rehabilitation}

Coventry et al recently conducted a systematic review with meta-analysis that examined the comparative effects of a broad range of psychological and/or lifestyle interventions on depression and anxiety in COPD. ${ }^{62}$ Interventions were divided into four subgroups: cognitive behavioral therapy (CBT) interventions, multicomponent interventions 
Table I Characteristics of the study populations

\begin{tabular}{|c|c|c|c|c|c|}
\hline Reference & $\begin{array}{l}\text { Sample } \\
\text { size }\end{array}$ & $\begin{array}{l}\text { Mean } \\
\text { age, years }\end{array}$ & $\begin{array}{l}\text { Males } \\
(\%)\end{array}$ & $\begin{array}{l}\text { COPD severity } \\
\text { (GOLD stage) }\end{array}$ & $\begin{array}{l}\text { Where } \\
\text { recruited }\end{array}$ \\
\hline $\begin{array}{l}\text { Blumenthal } \\
\text { et al }{ }^{86}\end{array}$ & 158 & 50 & 44 & Severe (stage 3) & Secondary care \\
\hline Bucknall et al ${ }^{87}$ & 464 & 69.1 & 37 & $\begin{array}{l}\text { I, severe (stage } 3 \text { ) } \\
\text { C, severe (stage } 3 \text { ) }\end{array}$ & Secondary care \\
\hline de Blok et al ${ }^{88}$ & 21 & 64.1 & 43 & $\begin{array}{l}\text { I, moderate (stage } 2 \text { ) } \\
\mathrm{C}, \text { severe (stage } 3 \text { ) }\end{array}$ & Tertiary care \\
\hline $\begin{array}{l}\text { de Godoy } \\
\text { and de Godoy }\end{array}$ & 30 & 60.5 & 73 & Severe (stage 3) & Secondary care \\
\hline $\begin{array}{l}\text { Donesky- } \\
\text { Cuenco et al90 }\end{array}$ & 41 & 70 & 28 & $\begin{array}{l}\text { I, moderate (stage } 2 \text { ) } \\
\mathrm{C}, \text { severe (stage } 3 \text { ) }\end{array}$ & Primary care \\
\hline Effing et $\mathrm{al}^{91}$ & 142 & 63.4 & 59 & $\begin{array}{l}\text { I, moderate (stage } 2) \\
\mathrm{C} \text {, severe (stage } 3 \text { ) }\end{array}$ & Secondary care \\
\hline Elçi et al ${ }^{92}$ & 78 & 58.9 & 85 & Severe (stage 3) & Tertiary care \\
\hline Emery et $\mathrm{a}^{93}$ & 79 & 66.6 & 47 & Severe (stage 3) & Primary care \\
\hline Gift et $\mathrm{al}^{94}$ & 26 & 68.5 & 31 & Moderate (stage 2) & Primary care \\
\hline Griffiths et $\mathrm{al}^{95}$ & 200 & 68.3 & 60 & Severe (stage 3) & $\begin{array}{l}\text { Primary care and } \\
\text { secondary care }\end{array}$ \\
\hline Güell et al ${ }^{96}$ & 40 & 67 & 94 & Severe (stage 3) & Tertiary care \\
\hline Hospes et al ${ }^{97}$ & 39 & 62.2 & 60 & Moderate (stage 2) & Secondary care \\
\hline Hynninen et al ${ }^{98}$ & 51 & 61 & 49 & Moderate (stage 2) & Secondary care \\
\hline Kapella et al ${ }^{99}$ & 23 & 63 & 83 & $\begin{array}{l}\text { I, moderate (stage 2) } \\
\mathrm{C} \text {, moderate (stage 2) }\end{array}$ & Community \\
\hline Kayahan et al ${ }^{100}$ & 45 & 66 & 87 & Moderate (stage 2) & Tertiary care \\
\hline Kunik et al ${ }^{\mid 01}$ & 53 & 71.3 & 83 & Severe (stage 3) & Secondary care \\
\hline Kunik et al $\left.\right|^{102}$ & 238 & 66.3 & 97 & Severe (stage 3) & Primary care \\
\hline Lamers et al ${ }^{103}$ & 187 & 71 & 60 & $\begin{array}{l}\text { Mild to moderate } \\
\text { (stage I to } 2 \text { ) }\end{array}$ & Primary care \\
\hline Livermore et al ${ }^{104}$ & 41 & 73.4 & 44 & Moderate (stage 2) & Secondary care \\
\hline Lolak et al ${ }^{105}$ & 83 & 67.7 & 37 & Severe (stage 3) & Secondary care \\
\hline Lord et al ${ }^{106}$ & 28 & 67.4 & $\begin{array}{l}\text { Not } \\
\text { stated }\end{array}$ & Severe (stage 3) & Secondary care \\
\hline McGeoch et al ${ }^{107}$ & 159 & 71 & 59.5 & Moderate (stage 2) & Primary care \\
\hline Özdemir et al ${ }^{108}$ & 50 & 62.5 & 100 & Moderate (stage 2) & Tertiary care \\
\hline Paz-Díaz et al ${ }^{109}$ & 24 & 64.5 & 73 & Severe (stage 3) & Secondary care \\
\hline Ries et al $\left.\right|^{114}$ & 119 & 62.6 & 73 & Severe (stage 3) & Primary care \\
\hline $\begin{array}{l}\text { Sassi-Dambron } \\
\text { et al }{ }^{110}\end{array}$ & 89 & 67.4 & 55 & Moderate (stage 2) & Secondary care \\
\hline Spencer et al ${ }^{\prime \prime \prime}$ & 59 & 66 & 46 & Moderate (stage 2) & Secondary care \\
\hline
\end{tabular}




\begin{tabular}{|c|c|c|c|c|c|}
\hline $\begin{array}{l}\text { Depressed } \\
\text { at baseline }\end{array}$ & $\begin{array}{l}\text { Anxious } \\
\text { at baseline }\end{array}$ & $\begin{array}{l}\text { Depression } \\
\text { assessment }\end{array}$ & $\begin{array}{l}\text { Anxiety } \\
\text { assessment }\end{array}$ & $\begin{array}{l}\text { Baseline mean (SD) } \\
\text { depression score }\end{array}$ & $\begin{array}{l}\text { Baseline mean (SD) } \\
\text { anxiety score }\end{array}$ \\
\hline \multirow[t]{2}{*}{ No } & No & $\mathrm{BDI}$ & STAI & I, I $3.4(8.3)$ & I, $40.3(12.6)$ \\
\hline & & & & C, $10.9(7.4)$ & C, $35.6(11.3)$ \\
\hline \multirow[t]{2}{*}{ Yes } & Yes & HADS & HADS & I, 8.5 (3.9) & I, IO (4.5) \\
\hline & & & & C, $8.3(4.1)$ & C, $9.3(4.6)$ \\
\hline \multirow[t]{2}{*}{ No } & No & $\mathrm{BDI}$ & N/A & I, I2.6 (95\% Cl 7.5-I7.7) & $\mathrm{N} / \mathrm{A}$ \\
\hline & & & & C, $12.9(95 \% \mathrm{Cl} 8.5-17.2)$ & \\
\hline \multirow[t]{2}{*}{ Yes } & Yes & $\mathrm{BDI}$ & BAI & I, I $3.7(8.9)$ & I, I $2.9(6.9)$ \\
\hline & & & & C, I4.9 (II.5) & C, $10.9(9.8)$ \\
\hline \multirow[t]{2}{*}{ No } & No & CES-D & STAI & I, $9.5(4.5)$ & I, $30.2(8)$ \\
\hline & & & & C, $12.6(9.4)$ & C, 33.8 (9) \\
\hline \multirow[t]{2}{*}{ No } & No & HADS & HADS & I, $4.4(3.5)$ & I, $4.6(3.3)$ \\
\hline & & & & C, $4.6(4)$ & C, $4.8(4)$ \\
\hline No & No & HADS & HADS & Not reported & Not reported \\
\hline \multirow[t]{3}{*}{ No } & No & SCL-depression & SCL-anxiety & I, $59.2(7.6)^{\mathrm{a}}$ & I, $54.3(7.2)^{\mathrm{a}}$ \\
\hline & & & & I, $55.5(5.3)^{\mathrm{b}}$ & I, $54.0(5.3)^{\mathrm{b}}$ \\
\hline & & & & C, 60 (7.7) & C, $53.4(4.5)$ \\
\hline \multirow[t]{2}{*}{ No } & No & $\mathrm{N} / \mathrm{A}$ & STAI & N/A & I, 45 (9) \\
\hline & & & & & C, 37 (6) \\
\hline \multirow[t]{2}{*}{ No } & No & HADS & HADS & I, 7.3 (3.2) & I, 8.6 (4.7) \\
\hline & & & & C, $7.5(4.3)$ & C, $8.9(4.3)$ \\
\hline \multirow[t]{2}{*}{ No } & No & SCL-90-R & SCL-90-R & I, I.3 (0.8) & I, I.0 (0.5) \\
\hline & & & & C, $0.6(0.6)$ & C, $0.6(0.7)$ \\
\hline \multirow[t]{2}{*}{ No } & No & BDI & $\mathrm{N} / \mathrm{A}$ & I, 8.4 (5.2) & $\mathrm{N} / \mathrm{A}$ \\
\hline & & & & C, 9.1 (8.3) & \\
\hline \multirow[t]{2}{*}{ Yes } & Yes & BDI-II & BAI & I, $20.7(8.6)$ & I, I7.5 (7.3) \\
\hline & & & & C, $20.5(9.7)$ & C, 17.5 (9.5) \\
\hline \multirow[t]{2}{*}{ Unknown } & Unknown & POMS-D & POMS-A & I, 9.9 (I0.3) & I, 9.4 (8.2) \\
\hline & & & & C, $10.4(8.2)$ & C, 8.6 (3.7) \\
\hline \multirow[t]{2}{*}{ No } & No & HAM-D & HAM-A & I, $5.43(4.8)$ & I, 8.9| (6.9) \\
\hline & & & & C, $7.18(6.5)$ & C, $7.91(6.6)$ \\
\hline \multirow[t]{2}{*}{ No } & No & GDS & BAI & I, I I.5 (0.3) & I, I5.3 (9.2) \\
\hline & & & & C, $7.7(5.4)$ & C, $10(6.8)$ \\
\hline \multirow[t]{2}{*}{ Yes } & Yes & BDI-II & BAI & I, 23.4 (I2.5) & I, 22.67 (I4.2) \\
\hline & & & & C, $21 . I(12)$ & C, 23 (I3.9) \\
\hline \multirow[t]{2}{*}{ Yes } & No & BDI-II & $\mathrm{SCL}$ & I, I7.I (6.5) & I, $20.6(6.2)$ \\
\hline & & & & C, I8.3 (7.2) & C, $20.4(7.3)$ \\
\hline \multirow[t]{2}{*}{ No } & No & HADS & HADS & I, $3.9(2.1)$ & I, $5.2(2.9)$ \\
\hline & & & & C, 4.1 (2.8) & C, 5.9 (2.7) \\
\hline \multirow[t]{2}{*}{ No } & No & HADS & HADS & I, 6.6 (4) & $\mathrm{T}, 6(4.3)$ \\
\hline & & & & C, 4.9 (3) & C, 6.35 (3.8) \\
\hline No & No & HADS & HADS & I, 5.7 (2.8) & I, $6.3(3.1)$ \\
\hline & & & & C, $5.8(3.6)$ & C, $5.3(2.6)$ \\
\hline No & No & HADS & HADS & I, 4.6 (3.7) & I, 6.2 (4.2) \\
\hline & & & & C, 4.1 (2.9) & C, 5.3 (3.6) \\
\hline No & No & HADS & HADS & I, 6 (3) & I, 6.8 (3.2) \\
\hline & & & & C, $7.0(4.6)$ & C, 7.I (4.9) \\
\hline No & No & BDI & STAI & I, I 4 (8) & I, 35 (26) \\
\hline & & & & C, 18 (8) & C, 33 (25) \\
\hline No & No & CES-D & $\mathrm{N} / \mathrm{A}$ & I, I4.0 (8.7) & $\mathrm{N} / \mathrm{A}$ \\
\hline & & & & C, I5.3 (10) & \\
\hline No & No & CES-D & STAI & I, I4.2 (I0.2) & I, 33.8 (9.7) \\
\hline & & & & C, $11.9(7.6)$ & C, 34.I (9.5) \\
\hline No & No & HADS & HADS & $\mathrm{I}, 4$ (2) & I, 6 (3) \\
\hline & & & & C, 5 (3) & C, 6 (3) \\
\hline
\end{tabular}


Table I (Continued)

\begin{tabular}{|c|c|c|c|c|c|}
\hline Reference & $\begin{array}{l}\text { Sample } \\
\text { size }\end{array}$ & $\begin{array}{l}\text { Mean } \\
\text { age, years }\end{array}$ & $\begin{array}{l}\text { Males } \\
(\%)\end{array}$ & $\begin{array}{l}\text { COPD severity } \\
\text { (GOLD stage) }\end{array}$ & $\begin{array}{l}\text { Where } \\
\text { recruited }\end{array}$ \\
\hline Taylor et al ${ }^{\prime \prime 2}$ & 116 & 69.5 & 46 & Moderate (stage 2) & Primary care \\
\hline Yeh et $\mathrm{al}^{113}$ & 10 & 65.5 & 60 & Moderate (stage 2) & Secondary care \\
\hline $\begin{array}{l}\text { Alexopoulos } \\
\text { et al }{ }^{68}\end{array}$ & 138 & 68.5 & $\begin{array}{l}\text { Not } \\
\text { stated }\end{array}$ & Severe (stage 3 ) & Tertiary care \\
\hline Gurgun et $\mathrm{a}^{65}$ & 46 & 64.7 & 95.6 & Severe (stage 3) & Tertiary care \\
\hline \multirow[t]{4}{*}{ Jiang et $\mathrm{al}^{69}$} & 100 & 64.9 & 69.75 & Control: moderate (stage 2) & Tertiary care \\
\hline & & 5 & & $63.8 \%$; severe (stage 3) $36.2 \%$ & \\
\hline & & & & Intervention: moderate (stage 2) & \\
\hline & & & & $59.2 \%$; severe (stage 3) $40.8 \%$ & \\
\hline Wadell et $\mathrm{a}^{66}$ & 48 & 55.8 & 56 & Severe (stage 3 ) & Tertiary care \\
\hline Walters et $\mathrm{al}^{67}$ & 182 & 67 & 52.5 & Moderate (stage 2) & Primary care \\
\hline
\end{tabular}

Notes: ${ }^{a}$ Comparison I, exercise, education, and stress management. ${ }^{\circ}$ Comparison 2, education and stress management.

Abbreviations: BAI, Beck Anxiety Inventory; BDI, Beck Depression Inventory; CES-D, Centre for Epidemiologic Studies Depression Scale; C, Control group; Cl, confidence interval; COPD, chronic obstructive pulmonary disease; GDS, Geriatric Depression Scale; GOLD, Global Initiative for Chronic Obstructive Lung Disease; HADS, Hospital Anxiety and Depression Scale; HAM-A, Hamilton Anxiety Rating Scale; HAM-D, Hamilton Depression Rating Scale; I, intervention group; N/A, not applicable; POMS-A, Profile of Mood States Anxiety scale; POMS-D, Profile of Mood States Depression scale; SCL, Symptom Checklist; SCL-90-R, Symptom Checklist-90-Revised; SD, standard deviation; STAI, State Trait Anxiety Inventory.

with an exercise component, relaxation techniques, and self-management education. This meta-analysis included 29 randomized controlled trials and 2,063 participants, and demonstrated that the pooled effects of psychological and/or lifestyle interventions led to small but significant reductions in symptoms of depression (standardized mean difference [SMD] $0.28,95 \% \mathrm{CI}-0.41,-0.14)$ and anxiety (SMD $-0.23,95 \% \mathrm{CI}-0.38,-0.09$ ). When grouped according to intervention components, the only intervention associated with significant improvements in symptoms of depression (SMD $-0.47,95 \% \mathrm{CI}-0.66,-0.28$ ) and anxiety (SMD $-0.45,95 \%$ CI $-0.71,-0.18$ ) was multicomponent pulmonary rehabilitation. Cognitive and behavioral treatment approaches and relaxation techniques were associated with small but not significant reductions in depression and anxiety. Self-management interventions that included disease education did not have an effect on depression or anxiety symptoms.

When the analysis was restricted to the five trials that included both psychological and exercise components, the effect size increased to 0.64 for depression and to 0.59 for anxiety, suggesting that complex interventions containing a combination of psychological techniques and exercise training have the greatest effects on depression and anxiety. ${ }^{62}$

This meta-analysis observed a great variability in the methods used to assess depression and anxiety across the studies included in the meta-analysis; some of the studies included patients with a diagnosis of depression and anxiety, while others measured symptoms of depression and anxiety (some of which did not report above threshold levels of depression). Coventry et al showed that the effectiveness of psychological and/or lifestyle interventions for reducing symptoms of depression and anxiety is equivalent across studies with confirmed depressed or above threshold samples (SMD -0.29 and -0.21 for depression and anxiety, respectively) and studies with unknown levels of depression and anxiety at baseline (SMD -0.24 and -0.27 for depression and anxiety, respectively). ${ }^{62}$ Better reporting of severity of depression at baseline in clinical trials will aid more informed assessment of the impact of symptom severity on treatment outcomes.

\section{Updated systematic review}

In recognition of the expanding evidence base and the clinical importance of this area, we updated the systematic review completed by Coventry et al in $2013 .{ }^{62}$ 


\begin{tabular}{|c|c|c|c|c|c|}
\hline $\begin{array}{l}\text { Depressed } \\
\text { at baseline }\end{array}$ & $\begin{array}{l}\text { Anxious } \\
\text { at baseline }\end{array}$ & $\begin{array}{l}\text { Depression } \\
\text { assessment }\end{array}$ & $\begin{array}{l}\text { Anxiety } \\
\text { assessment }\end{array}$ & $\begin{array}{l}\text { Baseline mean (SD) } \\
\text { depression score }\end{array}$ & $\begin{array}{l}\text { Baseline mean (SD) } \\
\text { anxiety score }\end{array}$ \\
\hline \multirow[t]{2}{*}{ No } & No & HADS & HADS & $\mathrm{I}, 5.4$ & $\mathrm{I}, 6 . \mathrm{I}$ \\
\hline & & & & C, 4.8 & $C, 6.7$ \\
\hline \multirow[t]{3}{*}{ No } & No & CES-D & $\mathrm{N} / \mathrm{A}$ & I, I4 (II-46) & $\mathrm{N} / \mathrm{A}$ \\
\hline & & & & C, $12(2-17)$ & \\
\hline & & & & (median, range) & \\
\hline \multirow[t]{2}{*}{ Yes } & N/A & HAM-D & N/A & I, $24.72(3.86)$ & N/A \\
\hline & & & & C, $24.80(3.46)$ & \\
\hline \multirow[t]{3}{*}{ No } & No & HADS & HADS & I, $8.4(3.1)^{\mathrm{a}}$ & I, $9.1(5.6)^{\mathrm{a}}$ \\
\hline & & & & I, $6.8(3.6)^{\mathrm{b}}$ & I, $6.8(4.9)^{\mathrm{b}}$ \\
\hline & & & & C, $8.8(4.5)$ & C, $8.8(4.5)$ \\
\hline \multirow[t]{3}{*}{ No } & No & HADS & STAI & I, 7.16 (3.02) & Trait anxiety: \\
\hline & & & & C, $7.08(2.92)$ & I, 42.9I (6.78) \\
\hline & & & & & C, $42.46(7.04)$ \\
\hline \multirow[t]{2}{*}{ No } & No & HADS & HADS & I, 5. I (3.3) & I, 5.8 (3.5) \\
\hline & & & & C, $4.2(2.9)$ & C, 4.5 (2.8) \\
\hline \multirow[t]{4}{*}{ No } & No & HADS, CES-D & HADS & HADS: I, 4.6 (3.I) & I, 6.7 (4.I) \\
\hline & & & & C, $5 . I(3.6)$ & C, 7 (4.I) \\
\hline & & & & CES-D: I, 4.6 (3.I) & \\
\hline & & & & C, $5.1(3.6)$ & \\
\hline
\end{tabular}

\section{Methods}

The methods used to search, select, extract, and analyze data resembled that reported in the original systematic review. ${ }^{62}$ To avoid repetition, we will only briefly present some key methodological aspects of this updated systematic review.

\section{Data sources and search strategy}

All searches were initially carried out from inception to April $2012^{62}$ and were updated in April 2014. The following electronic databases were searched: Medline, Embase, PsycINFO, Cinahl, Web of Science, and Scopus. The above searches were complemented by hand searches of the reference lists of the included studies.

\section{Eligibility criteria}

Studies had to fulfill the following criteria to be included in the review (see Coventry et $\mathrm{al}^{62}$ for more details):

- Study design - cluster or individual randomized controlled trials

- Population - individuals with COPD confirmed by postbronchodilator spirometry of forced expiratory volume in 1 second/forced vital capacity ratio of $70 \%$, and a forced expiratory volume in 1 second of $80 \%$
- Intervention - single or multiple component interventions that include psychological and/or lifestyle components

- Comparators - any control (eg, waiting list, usual care, attention or active control)

- Outcomes - standardized measure of depression and/or anxiety.

\section{Study selection and data extraction}

The titles/abstracts and the full texts of potentially relevant studies were screened by four reviewers independently. Data were extracted using a standardized data extraction form. Extracted data included characteristics of patients, interventions, outcomes, and quality appraisal of the studies. Study authors were contracted to retrieve data not available in published reports. Any disagreements during the process of study selection and data extraction were resolved by consensus in group meetings with all review authors.

\section{Data analysis}

Meta-analyses using random effects models were undertaken to assess the effectiveness of different types of complex interventions on reducing symptoms of depression and anxiety in those with COPD. Effect sizes were expressed 
Table 2 Characteristics of the interventions

\begin{tabular}{|c|c|c|c|c|}
\hline Reference & Intervention & Control group & $\begin{array}{l}\text { Lifestyle } \\
\text { components }\end{array}$ & $\begin{array}{l}\text { Psychological } \\
\text { components }\end{array}$ \\
\hline $\begin{array}{l}\text { Alexopoulos } \\
\text { et al }{ }^{68}\end{array}$ & Problem-solving techniques & Usual care & Education & Problem-solving techniques \\
\hline $\begin{array}{l}\text { Blumenthal } \\
\text { et al }{ }^{86}\end{array}$ & $\begin{array}{l}\text { Telephone-based } \\
\text { coping skills training }\end{array}$ & $\begin{array}{l}\text { Usual medical care including } \\
\text { clinic visits with pulmonologists } \\
\text { and regular contact with nurse } \\
\text { coordinators }\end{array}$ & $\begin{array}{l}\text { General education } \\
\text { Relapse prevention }\end{array}$ & $\begin{array}{l}\text { Problem-solving techniques } \\
\text { CBT relaxation }\end{array}$ \\
\hline Bucknall et $\mathrm{a}^{87}$ & Supported self-management & $\begin{array}{l}\text { Usual medical care from GP } \\
\text { and hospital based specialists } \\
\text { (including out of hours care) }\end{array}$ & $\begin{array}{l}\text { General education, } \\
\text { skills training }\end{array}$ & $\begin{array}{l}\text { Miscellaneous } \\
\text { (empowerment and } \\
\text { increased self-efficacy) }\end{array}$ \\
\hline de Blok et al ${ }^{88}$ & $\begin{array}{l}\text { PR plus physical activity } \\
\text { counseling }\end{array}$ & $\begin{array}{l}\text { Regular PR containing exercise } \\
\text { training, dietary intervention } \\
\text { and educational modules }\end{array}$ & $\begin{array}{l}\text { General education } \\
\text { Exercise skills training, } \\
\text { behavior therapy }\end{array}$ & $\begin{array}{l}\text { Biofeedback miscellaneous } \\
\text { (physical activity counselling, } \\
\text { motivational interviewing) }\end{array}$ \\
\hline $\begin{array}{l}\text { de Godoy } \\
\text { and de Godoy }{ }^{89}\end{array}$ & $\begin{array}{l}\text { CBT, physiotherapy, } \\
\text { exercise and education }\end{array}$ & $\begin{array}{l}\text { Physiotherapy, exercise, } \\
\text { and education }\end{array}$ & $\begin{array}{l}\text { General education } \\
\text { Exercise } \\
\text { Skills training }\end{array}$ & $\begin{array}{l}\text { CBT relaxation } \\
\text { Miscellaneous (logotherapy) }\end{array}$ \\
\hline $\begin{array}{l}\text { Donesky- } \\
\text { Cuenco } \\
\text { et al } 2009^{90}\end{array}$ & Yoga training & $\begin{array}{l}\text { Usual care (also received } \\
\text { educational pamphlet, offered yoga } \\
\text { at the end as waiting list control) }\end{array}$ & $\begin{array}{l}\text { Exercise } \\
\text { Skills training }\end{array}$ & Miscellaneous (relaxation) \\
\hline Effing et $\mathrm{al}^{91}$ & $\begin{array}{l}\text { Psychotherapeutic exercise; } \\
\text { self- management education }\end{array}$ & Self-management education & $\begin{array}{l}\text { General education } \\
\text { Skills training } \\
\text { Exercise }\end{array}$ & Problem-solving techniques \\
\hline Elçi et $\mathrm{al}^{92}$ & PR & $\begin{array}{l}\text { Standard medical care } \\
\text { (including instructions on use } \\
\text { of respiratory medicines) }\end{array}$ & $\begin{array}{l}\text { General education } \\
\text { Exercise } \\
\text { Skills training }\end{array}$ & $\begin{array}{l}\text { Miscellaneous } \\
\text { (psychological counseling) }\end{array}$ \\
\hline Emery et $\mathrm{a}^{93}$ & $\begin{array}{l}\text { Treatment } \\
\text { a. Exercise, education } \\
\text { and stress management } \\
\text { Treatment } \\
\text { b. Education and stress } \\
\quad \text { management }\end{array}$ & Waiting list control & $\begin{array}{l}\text { General education } \\
\text { Group discussion } \\
\text { Exercise }\end{array}$ & $\begin{array}{l}\text { CBT relaxation } \\
\text { Miscellaneous } \\
\text { (stress management) }\end{array}$ \\
\hline Gift et $\mathrm{al}^{94}$ & $\begin{array}{l}\text { Progressive muscle relaxation } \\
\text { with prerecorded tapes }\end{array}$ & $\begin{array}{l}\text { Participants instructed to } \\
\text { sit quietly for } 20 \text { minutes }\end{array}$ & $\mathrm{N} / \mathrm{A}$ & $\begin{array}{l}\text { Relaxation (Bernstein and } \\
\text { Borkovec method) }\end{array}$ \\
\hline Griffiths et $\mathrm{al}^{95}$ & Multidisciplinary PR & Standard medical management & $\begin{array}{l}\text { General education } \\
\text { Exercise } \\
\text { Skills training }\end{array}$ & $\begin{array}{l}\text { Relaxation miscellaneous } \\
\text { (stress management to } \\
\text { promote mastery and } \\
\text { control over illness) }\end{array}$ \\
\hline Güell et al ${ }^{96}$ & $\begin{array}{l}\text { PR including breathing } \\
\text { training and exercise }\end{array}$ & Usual care & $\begin{array}{l}\text { General education } \\
\text { Exercise } \\
\text { Skills training }\end{array}$ & Relaxation \\
\hline Gurgun et $\mathrm{al}^{65}$ & $\begin{array}{l}\text { PR with exercise, education } \\
\text { and nutritional supplementation }\end{array}$ & Usual care & Exercise, education & Relaxation \\
\hline Hospes et a $\left.\right|^{97}$ & $\begin{array}{l}\text { Pedometer-based exercise } \\
\text { counseling program }\end{array}$ & Usual care & Exercise & $\begin{array}{l}\text { Biofeedback problem- } \\
\text { solving techniques } \\
\text { Exercise counseling } \\
\text { Motivational interviewing }\end{array}$ \\
\hline Hynninen et $\mathrm{al}^{98}$ & CBT & $\begin{array}{l}\text { Enhanced standard care } \\
\text { for COPD }\end{array}$ & $\mathrm{N} / \mathrm{A}$ & CBT \\
\hline Jiang et al ${ }^{69}$ & $\begin{array}{l}\text { Uncertainty management } \\
\text { with } C B T\end{array}$ & Usual care & Skills training & $\mathrm{CBT}$, relaxation \\
\hline Kapella et al ${ }^{99}$ & CBT & COPD education & $\mathrm{N} / \mathrm{A}$ & CBT \\
\hline Kayahan et al ${ }^{100}$ & PR & Usual care & $\begin{array}{l}\text { General education } \\
\text { Exercise } \\
\text { Skills training }\end{array}$ & Relaxation \\
\hline
\end{tabular}




\begin{tabular}{|c|c|c|c|c|}
\hline Sessions (n) & $\begin{array}{l}\text { Session length } \\
\text { (minutes) }\end{array}$ & Delivered by & Delivery method & Follow-up \\
\hline 9 & $\begin{array}{l}30 \text { (for discharge } \\
\text { session) }\end{array}$ & Social workers & $\begin{array}{l}\text { Not reported (first session } \\
\text { was at discharge and remainder } \\
\text { in their own homes) }\end{array}$ & 28 weeks \\
\hline 12 & 30 & $\begin{array}{l}\text { Clinical psychologists, } \\
\text { social workers }\end{array}$ & $\begin{array}{l}\text { Individual, face-to-face, } \\
\text { and remote }\end{array}$ & 12 weeks \\
\hline 22 & 40 & Respiratory nurses & Individual, face-to-face & 52 weeks \\
\hline 4 & 30 & Physical therapists & $\begin{array}{l}\text { Group and individual, } \\
\text { face-to-face }\end{array}$ & 9 weeks \\
\hline $\begin{array}{l}24 \text { exercise sessions } \\
24 \text { physiotherapy sessions } \\
12 \text { psychotherapy sessions }\end{array}$ & Not reported & Respiratory physicians & Group, face-to-face & 12 weeks \\
\hline 24 & 60 & Expert yoga instructors & Group, face-to-face & 12 weeks \\
\hline $\begin{array}{l}\text { Four education sessions } \\
\text { First phase: } 72 \text { exercise sessions } \\
\text { Second phase: } 40 \text { voluntary } \\
\text { exercise sessions }\end{array}$ & $\begin{array}{l}\text { I } 20 \text { education } \\
\text { sessions }\end{array}$ & $\begin{array}{l}\text { Respiratory nurse } \\
\text { and physiotherapist }\end{array}$ & $\begin{array}{l}\text { Group, face-to-face, } \\
\text { and remote }\end{array}$ & 28 weeks \\
\hline 24 & 90 & Nurse & $\begin{array}{l}\text { Individual, face-to-face, } \\
\text { and remote }\end{array}$ & 4 weeks \\
\hline $\begin{array}{l}37 \text { exercise classes } \\
16 \text { lectures } \\
10 \text { stress management sessions }\end{array}$ & $\begin{array}{l}240 \\
\text { (all modules) }\end{array}$ & $\begin{array}{l}\text { Respiratory specialists } \\
\text { and clinical psychologist }\end{array}$ & Group, face-to-face & 10 weeks \\
\hline 4 & 20 & Primary care practitioners & Individual, face-to-face & 4 weeks \\
\hline 18 & 120 & $\begin{array}{l}\text { Occupational therapist, } \\
\text { physiotherapist, dietetic staff, } \\
\text { specialist respiratory nurse, and } \\
\text { a smoking cessation counselor }\end{array}$ & Group, face-to-face & 6 weeks \\
\hline $\begin{array}{l}\text { Phase I, } 16 \text { sessions } \\
\text { Phase } 2,40 \text { sessions }\end{array}$ & 30 & Not reported & Group, face-to-face & 16 weeks \\
\hline 16 & $60-80$ & Not stated & Not stated & 8 weeks \\
\hline 5 & 30 & Trained exercise counselor & Individual, face-to-face & 12 weeks \\
\hline 7 & 60 & $\begin{array}{l}\text { Masters level psychology } \\
\text { student }\end{array}$ & Group, face-to-face & 4 weeks \\
\hline 4 & 35 & Intervention nurses & Telephone & 40 weeks \\
\hline 6 & Not reported & $\begin{array}{l}\text { Nurse behavioral sleep } \\
\text { medicine specialist }\end{array}$ & Group, face-to-face & 6 weeks \\
\hline 24 & 150 & Not reported & $\begin{array}{l}\text { Individual and group, } \\
\text { face-to-face }\end{array}$ & 8 weeks \\
\hline
\end{tabular}


Table 2 (Continued)

\begin{tabular}{|c|c|c|c|c|}
\hline Reference & Intervention & Control group & $\begin{array}{l}\text { Lifestyle } \\
\text { components }\end{array}$ & $\begin{array}{l}\text { Psychological } \\
\text { components }\end{array}$ \\
\hline Kunik et al ${ }^{101}$ & CBT & COPD education & N/A & CBT \\
\hline Kunik et al ${ }^{102}$ & $\begin{array}{l}\text { CBT group treatment } \\
\text { intervention }\end{array}$ & COPD education & $\mathrm{N} / \mathrm{A}$ & CBT \\
\hline Lamers et al ${ }^{103}$ & $\begin{array}{l}\text { Minimal psychological } \\
\text { intervention }\end{array}$ & Usual care & Skills training & $\begin{array}{l}\text { Problem-solving } \\
\text { techniques CBT }\end{array}$ \\
\hline Livermore et al ${ }^{104}$ & $\mathrm{CBT}$ & Routine care (including PR) & $\mathrm{N} / \mathrm{A}$ & CBT \\
\hline Lolak et al ${ }^{105}$ & $\begin{array}{l}\text { Progressive muscle } \\
\text { relaxation and PR }\end{array}$ & Exercise training & $\begin{array}{l}\text { General education } \\
\text { Exercise } \\
\text { Skills training }\end{array}$ & $\begin{array}{l}\text { Relaxation (Bernstein } \\
\text { and Borkovec method) }\end{array}$ \\
\hline Lord et al ${ }^{106}$ & Singing teaching & Usual care & Skills training & Relaxation \\
\hline McGeoch et al ${ }^{107}$ & $\begin{array}{l}\text { Usual care and education } \\
\text { on the use of a written } \\
\text { self-management plan }\end{array}$ & Usual GP care & $\begin{array}{l}\text { General education } \\
\text { Skills training }\end{array}$ & N/A \\
\hline Özdemir et al ${ }^{108}$ & Water-based PR & Usual care & Exercise & N/A \\
\hline Paz-Díaz et al ${ }^{109}$ & $\begin{array}{l}\text { Exercise rehabilitation } \\
\text { program }\end{array}$ & Usual care & $\begin{array}{l}\text { Exercise } \\
\text { Skills training }\end{array}$ & $\begin{array}{l}\text { Miscellaneous (relaxation } \\
\text { techniques) }\end{array}$ \\
\hline Ries $^{1 / 4}$ & Pulmonary rehabilitation & $\begin{array}{l}\text { Education (videotapes, lectures, } \\
\text { and discussions but no individual } \\
\text { instruction or exercise training) }\end{array}$ & $\begin{array}{l}\text { General education } \\
\text { Exercise } \\
\text { Skills training }\end{array}$ & $\begin{array}{l}\text { Relaxation miscellaneous } \\
\text { (psychological support) }\end{array}$ \\
\hline $\begin{array}{l}\text { Sassi-Dambron } \\
\text { et al }{ }^{110}\end{array}$ & $\begin{array}{l}\text { Dyspnea self-management } \\
\text { training }\end{array}$ & General health education & $\begin{array}{l}\text { General education } \\
\text { Group discussion } \\
\text { Skills training }\end{array}$ & $\begin{array}{l}\text { Relaxation (progressive } \\
\text { muscle relaxation) } \\
\text { Miscellaneous (self-talk } \\
\text { and panic control) }\end{array}$ \\
\hline Spencer et al'"I & $\begin{array}{l}\text { Supervised outpatient-based } \\
\text { exercise plus unsupervised } \\
\text { home exercise }\end{array}$ & Unsupervised exercise & Exercise & $\mathrm{N} / \mathrm{A}$ \\
\hline Taylor et al $\left.\right|^{1 / 2}$ & $\begin{array}{l}\text { Disease-specific self- } \\
\text { management program }\end{array}$ & Usual care & Skills training & $\begin{array}{l}\text { Miscellaneous (self- } \\
\text { management using social } \\
\text { cognitive self-efficacy theory) }\end{array}$ \\
\hline Wadell et $a^{66}$ & PR & Usual care & Exercise, education & $\begin{array}{l}\text { Miscellaneous (managing } \\
\text { emotions and stress) }\end{array}$ \\
\hline Walters et $\mathrm{a}^{67}$ & $\begin{array}{l}\text { Health mentoring using } \\
\text { negotiated goal setting }\end{array}$ & Usual care & $\begin{array}{l}\text { Education, } \\
\text { skills training }\end{array}$ & $\begin{array}{l}\text { CBT, problem-solving } \\
\text { techniques }\end{array}$ \\
\hline Yeh et $\mathrm{al}^{113}$ & Tai Chi classes & Usual care & Exercise & $\begin{array}{l}\text { Relaxation miscellaneous } \\
\text { (meditation and mindfulness) }\end{array}$ \\
\hline
\end{tabular}

Abbreviations: CBT, cognitive and behavioral therapy; COPD, chronic obstructive pulmonary disease; GP, general practitioner; N/A, not applicable; PR, pulmonary rehabilitation.

as the SMD; an SMD of 0.56-1.2 is large, SMD 0.33-0.55 is moderate, and SMD of $<0.32$ is small. ${ }^{63}$ Heterogeneity was evaluated using the $I^{2}$, which provides a quantitative measure of the degree of between-study differences caused by factors other than sampling error; higher $I^{2}$ rates indicate higher heterogeneity. ${ }^{64}$

\section{Results}

The updated searches yielded 736 citations excluding duplicates. Of these, 714 citations were excluded at the title and abstract screening stage. The full texts for 22 citations were retrieved and checked against the eligibility criteria of the review. Following full-text screening, we identified five additional studies (providing six relevant comparisons) as eligible for inclusion in the review.

\section{Characteristics of included studies}

A total of 34 studies that provided 36 relevant comparisons $(n=2,577)$ were included in the updated meta-analysis. The COPD patients had a median age of 66 years with an equal sex distribution. The severity of COPD ranged from moderate to severe across the majority of the studies (see Table 1 for patient characteristics).

The majority of studies $(80 \%)$ evaluated complex interventions that included both psychological and lifestyle components, while six included only psychological components, 


\begin{tabular}{|c|c|c|c|c|}
\hline Sessions (n) & $\begin{array}{l}\text { Session length } \\
\text { (minutes) }\end{array}$ & Delivered by & Delivery method & Follow-up \\
\hline I (+6 phone calls) & 120 & $\begin{array}{l}\text { Board-certified } \\
\text { gero-psychiatrist }\end{array}$ & $\begin{array}{l}\text { Group, face-to-face } \\
\text { and individual, remote }\end{array}$ & 6 weeks \\
\hline 8 & 60 & $\begin{array}{l}\text { Psychology interns and } \\
\text { postdoctoral fellows }\end{array}$ & Group, face-to-face & 4 weeks \\
\hline Average of 4 contacts & 60 & Primary care nurses & Individual, face-to-face & 12 week \\
\hline 4 & 60 & Clinical psychologist & Individual, face-to-face & 6 weeks \\
\hline 12 & 60 & Multidisciplinary PR team & Group, face-to-face & 8 weeks \\
\hline 12 & 60 & Singing teacher & Group, face-to-face & 7 weeks \\
\hline I & 60 & $\begin{array}{l}\text { Practice nurse or respiratory } \\
\text { educator in association } \\
\text { with GP }\end{array}$ & Individual, face-to-face & 24 weeks \\
\hline 12 & 35 & $\begin{array}{l}\text { Physiotherapist } \\
\text { and chest physician }\end{array}$ & Group, face-to-face & 4 weeks \\
\hline 24 & 85 & Not reported & Group, face-to-face & 8 weeks \\
\hline 12 & 240 & Not reported & Group, face-to-face & 8 weeks \\
\hline 6 & Not reported & $\begin{array}{l}\text { Graduate student } \\
\text { in psychology and } \\
\text { a clinical nurse }\end{array}$ & Group, face-to-face & 6 weeks \\
\hline 52 & 50 & Physiotherapist & Group, face-to-face & 12 weeks \\
\hline 7 & 150 & $\begin{array}{l}\text { Lay trainer and } \\
\text { respiratory physician }\end{array}$ & Group, face-to-face & 8 weeks \\
\hline 24 & 210 & COPD nurse & Face-to-face & 8 weeks \\
\hline 16 & 30 & $\begin{array}{l}\text { Community health } \\
\text { nurses }\end{array}$ & Telephone & 24 weeks \\
\hline 24 & 60 & Tai Chi instructors & Group, face-to-face & 12 weeks \\
\hline
\end{tabular}

and four lifestyle interventions alone. Among the five trials identified from the new searches, two studies (including three comparisons) comprised multicomponent exercise interventions ${ }^{65,66}$ and three studies comprised CBT interventions. ${ }^{67-69}$ None of the new trials evaluated relaxation techniques or self-management interventions (see Table 2 for intervention characteristics).

\section{Effects of different types of complex interventions on depression and anxiety}

Thirty-four trials reported data on depression and 30 trials reported data on anxiety. As with the results of the original review, ${ }^{62}$ the pooled effects of the interventions indicated small but significant improvements in depression (SMD -0.30, 95\% $\mathrm{CI}-0.41,-0.19)$ and in anxiety (SMD $-0.31,95 \% \mathrm{CI}-0.49$, -0.10). Subgroup analysis showed that CBT interventions were associated with small and significant improvements in depression. The results for the subgroup of multicomponent exercise training interventions were unchanged; multicomponent exercise training interventions were associated with the largest treatment effects in favor of a reduction in depression and anxiety (forest plot, Figures 1 and 2).

\section{Implications for practice and research}

Multicomponent exercise training with or without psychological support is associated with the greatest improvements 


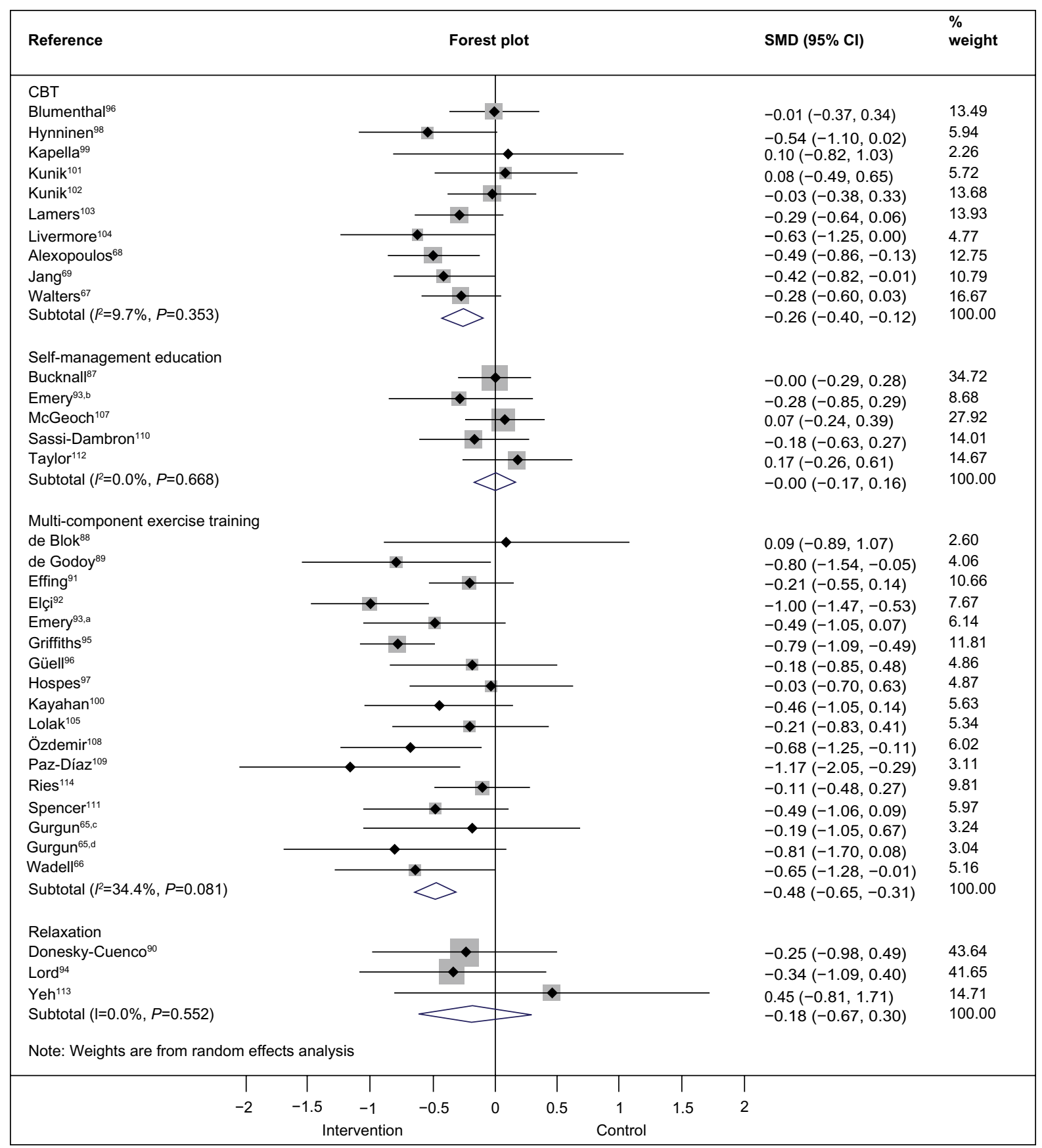

Figure I Effects of subgroups of complex interventions on self-reported depression at post-treatment.

Note: Random-effects model was used. alndependent comparison I, exercise, education, and stress management; bindependent comparison 2, education and stress management; 'independent comparison I, pulmonary rehabilitation and nutritional support; independent comparison 2, pulmonary rehabilitation.

Abbreviations: $\mathrm{CBT}$, cognitive and behavioral therapy; $\mathrm{Cl}$, confidence interval; SMD, standardized mean difference.

in symptoms of depression and anxiety in COPD compared with other nonpharmacological approaches. Components of pulmonary rehabilitation vary, but typically include prescribed supervised exercise training and self-management advice as well as multidisciplinary education about COPD and nutrition for a minimum of 6 weeks. Psychological and behavioral interventions may also be provided in the context of self-management advice, with an emphasis on promoting adaptive behaviors such as self-efficacy. ${ }^{51}$ However, psychological interventions are rarely provided alongside or integrated within pulmonary rehabilitation. ${ }^{70}$ Future research could address whether mental health professionals, in collaboration with multidisciplinary pulmonary rehabilitation teams, could play important roles in the delivery of psychological 


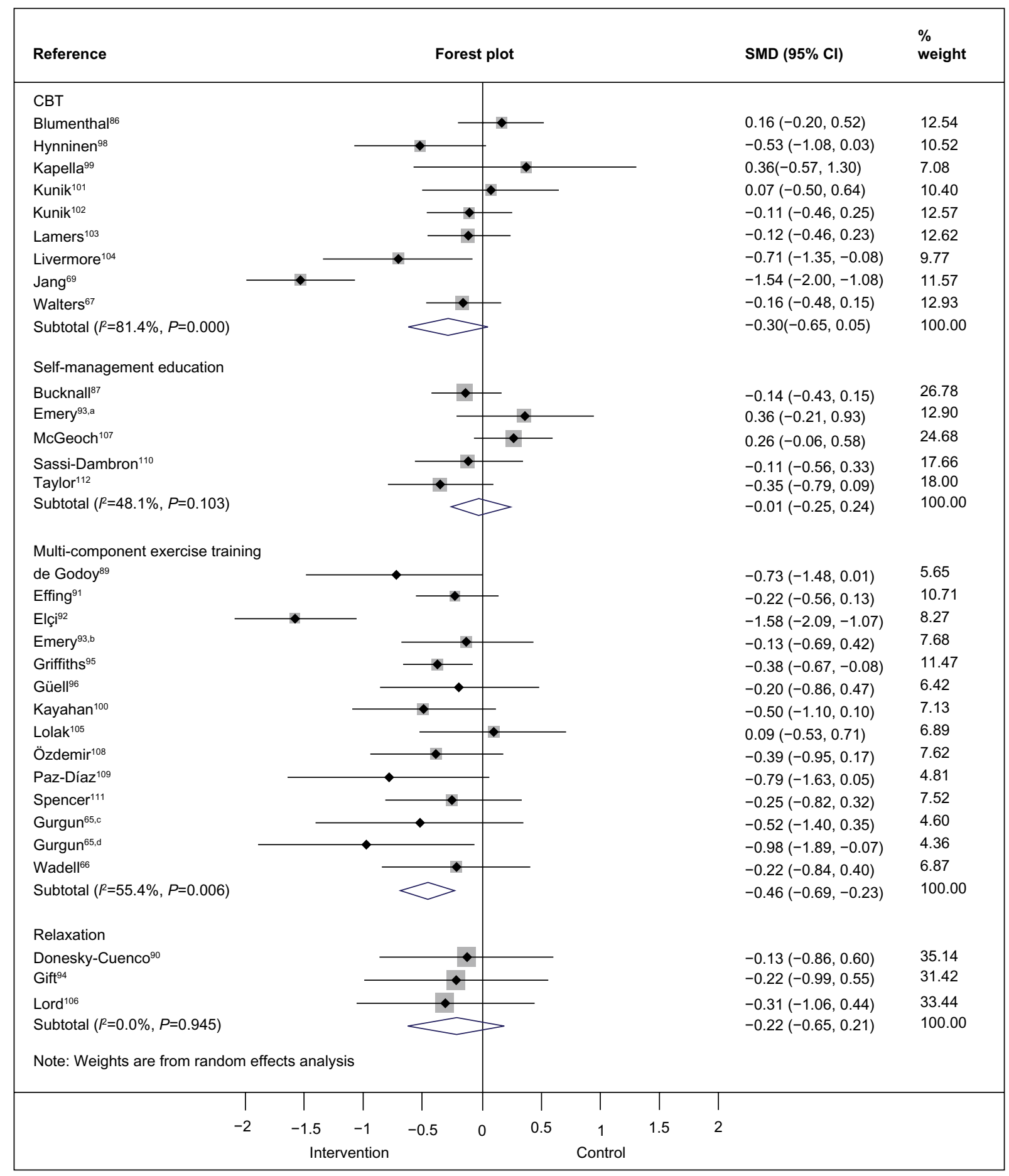

Figure 2 Effects of subgroups of complex interventions on self-reported anxiety at post-treatment.

Note: Random-effects model was used. aEducation and stress management; ' exercise, education, and stress management; 'independent comparison I, pulmonary rehabilitation and nutritional support; independent comparison 2, pulmonary rehabilitation.

Abbreviations: CBT, cognitive and behavioral therapy; $\mathrm{Cl}$, confidence interval; SMD, standardized mean difference.

interventions for common mental health problems in COPD patients attending pulmonary rehabilitation.

Interventions based on a CBT format are also potentially effective for managing depression in COPD. These results are consistent with other meta-analyses showing that psychological interventions that include CBT significantly reduce symptoms of depression in people with long-term conditions. ${ }^{71,72}$ However, the size of the treatment effects associated with CBT in populations with long-term conditions are small and possibly of trivial importance for patients. 
Existing evidence about the beneficial effects of CBT in anxiety disorders ${ }^{73}$ and in other long-term conditions ${ }^{74}$ implies that unique features of COPD might account for the relatively small treatment effects for CBT in this patient group. For instance, the use of CBT techniques to counter ruminative thinking and avoidance behaviors might not be acceptable to COPD patients when these behaviors are triggered as a response to real and meaningful COPD symptoms such as dyspnea. ${ }^{62}$ Alternative or "third wave" psychological therapies that target the process of thoughts (rather than their content, as in CBT) and help people to become aware of their thoughts and accept them in a nonjudgmental way are equally effective for depression as $\mathrm{CBT}^{75}$ Mindfulness meditation is associated with longer-term mental health benefits when compared with relaxation alone ${ }^{76}$ and is acceptable among people with long-term conditions, ${ }^{77}$ but its effectiveness among COPD patients has not yet been confirmed. ${ }^{78}$

Other explanations for why stand-alone interventions such as CBT may only confer modest benefits in people with COPD point to the need to embed psychological interventions within collaborative and multidisciplinary frameworks that promote proactive case management of patients and supervision of psychological therapists. Collaborative care is a complex intervention that typically involves a case manager working in conjunction with the patient's physician (usually their primary care physician), often with the support and supervision of a mental health specialist (a psychiatrist or psychologist). When compared with usual care, collaborative care is associated with significant improvement in depression and anxiety outcomes over the short-, medium-, and long-term. ${ }^{75}$ There is also evidence that collaborative care can improve both physical and mental health in people with long-term conditions. ${ }^{79}$ However, there is less evidence that collaborative interventions are effective in COPD, and trials to date have focused on self-management interventions to reduce exacerbations and improve medication adherence in acute illness, not on reducing depression or anxiety. ${ }^{80,81}$

In this overview, we have focused on the benefits of nonpharmacological interventions for the management of depression and anxiety in COPD. Psychological interventions are as effective as drug therapies for improving the psychological ill health of patients with COPD and are rated as preferable to drug therapies by patients. ${ }^{57,82}$ Additionally, psychological interventions with or without medication have been recommended for managing depression and anxiety in COPD. ${ }^{55}$ To date, the levels of evidence for the efficacy of pharmacological interventions in reducing depression and anxiety in COPD are limited. Two recent reviews suggested that no firm conclusions can be drawn about the effectiveness of antidepressants (selective serotonin reuptake inhibitors and tricyclic antidepressants) in reducing depression in COPD because there are only a small number of published trials in this area, many of which have important methodological limitations, such as small sample sizes, and high dropout rates. ${ }^{83,84}$

\section{Conclusion and future directions}

There is ample research evidence that depression and anxiety are important determinants of health outcomes and health care utilization in COPD. Health care policy has highlighted the need to manage depression and anxiety in long-term conditions, including COPD, but finding effective and innovative ways of implementing existing treatments remains a major challenge. Contemporary research suggests that complex psychological and/or lifestyle interventions which include a pulmonary rehabilitation component have the greatest effects on depression and anxiety in patients with COPD. However, further work is needed to understand how exercise improves anxious and depressed moods in COPD. Additionally, CBT appears to be effective in improving depression in COPD, but its benefits could be enhanced if embedded within collaborative care models that integrate physical and mental health care. Collaborative care models that focus on building partnerships between mental health and other professionals to foster integration of care for people with complex morbidities present a fruitful framework for the management of mental health in COPD. In particular, the integration of pulmonary rehabilitation and psychological therapies such as CBT has the potential to lead to significant patient benefits. Moreover, further research into ways to target markers of psychological health such as HRQoL could advance the clinical management of mental health in COPD.

In conclusion, finding ways to strengthen the delivery of effective mental health care within the context of innovative chronic disease management programs such as pulmonary rehabilitation in primary care offer opportunities to meet the challenge set out by the World Health Organization that there can be "no health without mental health". 85

\section{Acknowledgments}

PC is funded by the National Institute for Health Research Collaboration for Leadership in Applied Health Research and Care for Greater Manchester. The views expressed in this paper are those of the authors and not necessarily those of the National Institute for Health Research, National Health Service, or the Department of Health. We thank Liz Baker and Dr Cassandra Kenning for supporting searches and the 
data extraction included in the updated review presented in this paper.

\section{Disclosure}

The authors report no conflicts of interest in this work.

\section{References}

1. Ayuso-Mateos JL, Vazquez-Barquero JL, Dowrick C, et al. Depressive disorders in Europe: prevalence figures from the ODIN study. $\mathrm{Br} J$ Psychiatry. 2001;179:308-316.

2. Uher R, Payne JL, Pavlova B, Perlis RH. Major depressive disorder in DSM-5: implications for clinical practice and research of changes from DSM-IV. Depress Anxiety. 2014;31(6):459-471.

3. American Psychiatric Association. Diagnostic and Statistical Manual of Mental Disorders. 5th ed. Arlington, VA, USA: American Psychiatric Publishing; 2013.

4. Ferrari AJ, Charlson FJ, Norman RE, et al. Burden of depressive disorders by country, sex, age, and year: findings from the Global Burden of Disease Study 2010. PLoS Med. 2013;10(11):e1001547.

5. Kessler RC, Petukhova M, Sampson NA, Zaslavsky AM, Wittchen HU. Twelve-month and lifetime prevalence and lifetime morbid risk of anxiety and mood disorders in the United States. Int J Methods Psychiatr Res. 2012;21(3):169-184.

6. Tyrer P. The case for cothymia: mixed anxiety and depression as a single diagnosis. Br J Psychiatry. 2001;179:191-193.

7. Katon WJ. Epidemiology and treatment of depression in patients with chronic medical illness. Dialogues Clin Neurosci. 2011;13(1) $7-23$.

8. Moussavi S, Chatterji S, Verdes E, Tandon A, Patel V, Ustun B. Depression, chronic diseases, and decrements in health: results from the World Health Surveys. Lancet. 2007;370(9590):851-858.

9. Zhang MW, Ho RC, Cheung MW, Fu E, Mak A. Prevalence of depressive symptoms in patients with chronic obstructive pulmonary disease: a systematic review, meta-analysis and meta-regression. Gen Hosp Psychiatry. 2011;33(3):217-223.

10. van Ede L, Yzermans CJ, Brouwer HJ. Prevalence of depression in patients with chronic obstructive pulmonary disease: a systematic review. Thorax. 1999;54(8):688-692.

11. Mannino DM, Buist AS. Global burden of COPD: risk factors, prevalence, and future trends. Lancet. 2007;370(9589):765-773.

12. Kunik ME, Roundy K, Veazey C, et al. Surprisingly high prevalence of anxiety and depression in chronic breathing disorders. Chest. 2005;127(4):1205-1211.

13. Willgoss TG, Yohannes AM. Anxiety disorders in patients with COPD: a systematic review. Respir Care. 2013;58(5):858-866.

14. Livermore N, Sharpe L, McKenzie D. Panic attacks and panic disorder in chronic obstructive pulmonary disease: a cognitive behavioral perspective. Respir Med. 2010;104(9):1246-1253.

15. Wagena EJ, Arrindell WA, Wouters EF, van Schayck CP. Are patients with COPD psychologically distressed? Eur Respir J. 2005;26(2) 242-248.

16. Lou P, Zhu Y, Chen P, et al. Prevalence and correlations with depression, anxiety, and other features in outpatients with chronic obstructive pulmonary disease in China: a cross-sectional case control study. $B M C$ Pulm Med. 2012;12:53.

17. Hanania NA, Mullerova H, Locantore NW, et al. Determinants of depression in the ECLIPSE chronic obstructive pulmonary disease cohort. Am J Respir Crit Care Med. 2011;183(5):604-611.

18. Di Marco F, Verga M, Reggente M, et al. Anxiety and depression in COPD patients: The roles of gender and disease severity. Respir Med. 2006;100(10):1767-1774.

19. Laurin C, Lavoie KL, Bacon SL, et al. Sex differences in the prevalence of psychiatric disorders and psychological distress in patients with COPD. Chest. 2007;132(1):148-155.
20. Bhui K, Bhugra D, Goldberg D, Sauer J, Tylee A. Assessing the prevalence of depression in Punjabi and English primary care attenders: the role of culture, physical illness and somatic symptoms. Transcult Psychiatry. 2004;41(3):307-322.

21. Gater R, Tomenson B, Percival C, et al. Persistent depressive disorders and social stress in people of Pakistani origin and white Europeans in UK. Soc Psychiatry Psychiatr Epidemiol. 2009;44(3):198-207.

22. Weich S, Nazroo J, Sproston K, et al. Common mental disorders and ethnicity in England: the EMPIRIC study. Psychol Med. 2004;34(8): $1543-1551$.

23. Bakas T, McLennon SM, Carpenter JS, et al. Systematic review of health-related quality of life models. Health Qual Life Outcomes. 2012;10:134.

24. Martinez Frances ME, Perpina Tordera M, Belloch Fuster A, Martinez Moragon EM, Compte Torrero L. [Impact of baseline and induced dyspnea on the quality of life of patients with COPD.] Arch Bronconeumol. 2008;44(3):127-134. Spanish.

25. Balcells E, Gea J, Ferrer J, et al. Factors affecting the relationship between psychological status and quality of life in COPD patients. Health Qual Life Outcomes. 2010;8:108.

26. Tsiligianni IG, van der Molen T. A systematic review of the role of vitamin insufficiencies and supplementation in COPD. Respir Res. 2010;11:171.

27. Blakemore A, Dickens C, Guthrie E, et al. Depression and anxiety predict health-related quality of life in chronic obstructive pulmonary disease: systematic review and meta-analysis. Int $J$ Chron Obstruct Pulmon Dis. 2014;9:501-512.

28. Stein MB, Cox BJ, Afifi TO, Belik SL, Sareen J. Does co-morbid depressive illness magnify the impact of chronic physical illness? A population-based perspective. Psychol Med. 2006;36(5):587-596.

29. Dalal AA, Shah M, Lunacsek O, Hanania NA. Clinical and economic burden of depression/anxiety in chronic obstructive pulmonary disease patients within a managed care population. COPD. 2011;8(4):293-299.

30. Ng TP, Niti M, Tan WC, Cao Z, Ong KC, Eng P. Depressive symptoms and chronic obstructive pulmonary disease: effect on mortality, hospital readmission, symptom burden, functional status, and quality of life. Arch Intern Med. 2007;167(1):60-67.

31. Laurin C, Moullec G, Bacon SL, Lavoie KL. Impact of anxiety and depression on chronic obstructive pulmonary disease exacerbation risk. Am J Respir Crit Care Med. 2012;185(9):918-923.

32. Xu W, Collet JP, Shapiro S, et al. Independent effect of depression and anxiety on chronic obstructive pulmonary disease exacerbations and hospitalizations. Am J Respir Crit Care Med. 2008;178(9):913-920.

33. Coventry PA, Gemmell I, Todd CJ. Psychosocial risk factors for hospital readmission in COPD patients on early discharge services: a cohort study. BMC Pulm Med. 2011;11:49.

34. Dahlen I, Janson C. Anxiety and depression are related to the outcome of emergency treatment in patients with obstructive pulmonary disease. Chest. 2002;122(5):1633-1637.

35. Gudmundsson G, Gislason T, Janson C, et al. Risk factors for rehospitalisation in COPD: role of health status, anxiety and depression. Eur Respir J. 2005;26(3):414-419.

36. Hochlehnert A, Niehoff D, Wild B, Junger J, Herzog W, Lowe B. Psychiatric comorbidity in cardiovascular inpatients: costs, net gain, and length of hospitalization. J Psychosom Res. 2011;70(2):135-139.

37. Hutter N, Schnurr A, Baumeister H. Health care costs in patients with diabetes mellitus and comorbid mental disorders - a systematic review. Diabetologia. 2010;53(12):2470-2479.

38. Gilmer TP, O'Connor PJ, Rush WA, et al. Predictors of health care costs in adults with diabetes. Diabetes Care. 2005;28(1):59-64.

39. Simon GE, Katon WJ, Lin EH, et al. Cost-effectiveness of systematic depression treatment among people with diabetes mellitus. Arch Gen Psychiatry. 2007;64(1):65-72.

40. Ciechanowski PS, Katon WJ, Russo JE. Depression and diabetes: impact of depressive symptoms on adherence, function, and costs. Arch Intern Med. 2000;160(21):3278-3285. 
41. Naylor C, Parsonage M, McDaid D, Knapp M, Fossey M, Galea A. Long-Term Conditions and Mental Health. The Cost of Co-Morbidities. London, UK: The Kings Fund; 2012.

42. Howard C, Dupont S, Haselden B, Lynch J, Wills P. The effectiveness of a group cognitive-behavioural breathlessness intervention on health status, mood and hospital admissions in elderly patients with chronic obstructive pulmonary disease. Psychol Health Med. 2010;15(4): 371-385.

43. Mannino DM, Kiriz VA. Changing the burden of COPD mortality. Int J Chron Obstruct Pulmon Dis. 2006;1(3):219-233.

44. Esteban C, Quintana JM, Moraza J, et al. BODE-Index vs HADO-score in chronic obstructive pulmonary disease: which one to use in general practice? BMC Med. 2010;8:28

45. Groenewegen KH, Schols AM, Wouters EF. Mortality and mortalityrelated factors after hospitalization for acute exacerbation of COPD. Chest. 2003;124(2):459-467.

46. Almagro P, Calbo E, Ochoa de Echaguen A, et al. Mortality after hospitalization for COPD. Chest. 2002;121(5):1441-1448.

47. Fan VS, Ramsey SD, Giardino ND, et al. Sex, depression, and risk of hospitalization and mortality in chronic obstructive pulmonary disease. Arch Intern Med. 2007;167(21):2345-2353.

48. de Voogd JN, Wempe JB, Koëter GH, et al. Depressive symptoms as predictors of mortality in patients with COPD. Chest. 2009;135(3): 619-625.

49. Lou P, Chen P, Zhang P, et al. Effects of smoking, depression, and anxiety on mortality in COPD patients: a prospective study. Respir Care. 2014;59(1):54-61.

50. Roche N. Where current pharmacological therapies fall short in COPD: symptom control is not enough. Eur Respir Rev. 2007;16: 98-104.

51. Spruit MA, Singh SJ, Garvey C, et al. An Official American Thoracic Society/European Respiratory Society Statement: key concepts and advances in pulmonary rehabilitation. Am J Respir Crit Care Med. 2013;188(8):e13-e64

52. Prince M, Patel V, Saxena S, et al. No health without mental health. Lancet. 2007;370(9590):859-877.

53. National Institute for Health and Care Excellence. The Treatment and Management of Depression in Adults (Updated Edition). NICE Clinical Guidelines, No 90. Leicester, UK: British Psychological Society; 2010. Available from: http://www.ncbi.nlm.nih.gov/books/NBK63748/. Accessed September 19, 2014.

54. National Institute for Health and Care Excellence. Depression in adults with a chronic physical health problem. Treatment and management. London, UK: National Institute for Health and Care Excellence; 2009. Available from: http://www.nice.org.uk/guidance/cg91. Accessed September 19, 2014.

55. Department of Health. An outcomes strategy for people with chronic obstructive pulmonary disease (COPD) and asthma in England. London, UK: Department of Health; 2011. Available from: https://www.gov. uk/government/publications/an-outcomes-strategy-for-people-withchronic-obstructive-pulmonary-disease-copd-and-asthma-in-england. Accessed September 19, 2014.

56. Cuijpers P, Sijbrandij M, Koole SL, Andersson G, Beekman AT, Reynolds CF 3rd. The efficacy of psychotherapy and pharmacotherapy in treating depressive and anxiety disorders: a meta-analysis of direct comparisons. World Psychiatry. 2013;12(2):137-148.

57. McHugh RK, Whitton SW, Peckham AD, Welge JA, Otto MW. Patient preference for psychological vs pharmacologic treatment of psychiatric disorders: a meta-analytic review. J Clin Psychiatry. 2013;74(6): 595-602.

58. Coventry PA, Hays R, Dickens C, et al. Talking about depression: a qualitative study of barriers to managing depression in people with long term conditions in primary care. BMC Fam Pract. 2011;10:12.

59. Lacasse Y, Goldstein R, Lasserson TJ, Martin S. Pulmonary rehabilitation for chronic obstructive pulmonary disease. Cochrane Database Syst Rev. 2006;4:CD003793.
60. National Institute for Health and Care Excellence. Chronic obstructive pulmonary disease (update). Clinical guideline 101. London, UK: National Institute for Health and Care Excellence; 2010. Available from: http://www.nice.org.uk/guidance/cg101. Accessed September 19, 2014.

61. Coventry PA. Does pulmonary rehabilitation reduce anxiety and depression in chronic obstructive pulmonary disease? Curr Opin Pulm Med. 2009;15(2):143-149.

62. Coventry PA, Bower P, Keyworth C, et al. The effect of complex interventions on depression and anxiety in chronic obstructive pulmonary disease: systematic review and meta-analysis. PLoS One. 2013;8(4): e60532.

63. Lipsey MW, Wilson DB. The efficacy of psychological, educational, and behavioral treatment. Confirmation from meta-analysis. Am Psychol. 1993;48(12):1181-1209.

64. Higgins JP, Thompson SG, Deeks JJ, Altman DG. Measuring inconsistency in meta-analyses. BMJ. 2003;327(7414):557-560.

65. Gurgun A, Deniz S, Argin M, Karapolat H. Effects of nutritional supplementation combined with conventional pulmonary rehabilitation in muscle-wasted chronic obstructive pulmonary disease: a prospective, randomized and controlled study. Respirology. 2013;18(3): 495-500.

66. Wadell K, Webb KA, Preston ME, et al. Impact of pulmonary rehabilitation on the major dimensions of dyspnea in COPD. COPD. 2013;10(4):425-435.

67. Walters J, Cameron-Tucker H, Wills K, et al. Effects of telephone health mentoring in community-recruited chronic obstructive pulmonary disease on self-management capacity, quality of life and psychological morbidity: a randomised controlled trial. BMJ Open. 2013;3(9): e003097.

68. Alexopoulos GS, Kiosses DN, Sirey JA, et al. Personalised intervention for people with depression and severe COPD. Br J Psychiatry. 2013;202(3):235-236.

69. Jiang X, He G. Effects of an uncertainty management intervention on uncertainty, anxiety, depression, and quality of life of chronic obstructive pulmonary disease outpatients. Res Nurs Health. 2012;35(4): 409-418.

70. Roberts C, Stone R, Pursey N, Potter J, O'Reilly J. The national chronic obstructive pulmonary disease resources and outcomes project (NCROP). London, UK: 2010. Available from: https://www.rcplondon. ac.uk/sites/default/files/ncrop-re-survey-report-nov-2010_0.pdf. Accessed September 19, 2014.

71. Harkness E, Macdonald W, Valderas J, Coventry P, Gask L, Bower P. Identifying psychosocial interventions that improve both physical and mental health in patients with diabetes: a systematic review and metaanalysis. Diabetes Care. 2010;33(4):926-930.

72. Dickens C, Cherrington A, Adeyemi I, et al. Characteristics of psychological interventions that improve depression in people with coronary heart disease: a systematic review and meta-regression. Psychosom Med. 2013;75(2):211-221.

73. Butler AC, Chapman JE, Forman EM, Beck AT. The empirical status of cognitive-behavioral therapy: a review of meta-analyses. Clin Psychol Rev. 2006;26(1):17-31.

74. Beltman MW, Voshaar RC, Speckens AE. Cognitive-behavioural therapy for depression in people with a somatic disease: meta-analysis of randomised controlled trials. Br J Psychiatry. 2010;197(1):11-19.

75. Archer J, Bower P, Gilbody S, et al. Collaborative care for depression and anxiety problems. Cochrane Database Syst Rev. 2012;10:CD006525.

76. Jain S, Shapiro SL, Swanick S, et al. A randomized controlled trial of mindfulness meditation versus relaxation training: effects on distress, positive states of mind, rumination, and distraction. Ann Behav Med. 2007;33(1):11-21.

77. Keyworth C, Knopp J, Roughley K, Dickens C, Bold S, Coventry P. A Mixed-methods pilot study of the acceptability and effectiveness of a brief meditation and mindfulness intervention for people with diabetes and coronary heart disease. Behav Med. 2013;40(2):53-64. 
78. Mularski RA, Munjas BA, Lorenz KA, et al. Randomized controlled trial of mindfulness-based therapy for dyspnea in chronic obstructive lung disease. J Altern Complement Med. 2009;15(10):1083-1090.

79. Katon WJ, Lin EH, Von Korff M, et al. Collaborative care for patients with depression and chronic illnesses. $N$ Engl J Med. 2010;363(27): 2611-2620.

80. Fan VS, Gaziano JM, Lew R, et al. A comprehensive care management program to prevent chronic obstructive pulmonary disease hospitalizations: a randomized, controlled trial. Ann Intern Med. 2012;156(10):673-683.

81. Rice KL, Dewan N, Bloomfield HE, et al. Disease management program for chronic obstructive pulmonary disease: a randomized controlled trial. Am J Respir Crit Care Med. 2010;182(7):890-896.

82. Yohannes AM, Connolly MJ, Baldwin RC. A feasibility study of antidepressant drug therapy in depressed elderly patients with chronic obstructive pulmonary disease. Int J Geriatr Psychiatry. 2001;16(5): 451-454.

83. Fritzsche A, Clamor A, von Leupoldt A. Effects of medical and psychological treatment of depression in patients with COPD - a review. Respir Med. 2011;105(10):1422-1433.

84. Hegerl U, Mergl R. Depression and suicidality in COPD: understandable reaction or independent disorders? Eur Respir J. 2014;44(3): 734-743.

85. World Health Organization. Mental health: facing the challenges, building solutions. Report from the WHO European Ministerial Conference. Copenhagen, Denmark; 2005. Available from: http://www. euro.who.int/_data/assets/pdf_file/0008/96452/E87301.pdf. Accessed September 19, 2014.

86. Blumenthal JA, Babyak MA, Keefe FJ, et al. Telephone-based coping skills training for patients awaiting lung transplantation. $J$ Consult Clinical Psychol. 2006;74(3):535-544.

87. Bucknall CE, Miller G, Lloyd SM, et al. Glasgow supported selfmanagement trial (GSuST) for patients with moderate to severe COPD: randomised controlled trial. BMJ. 2012;344:e1060.

88. de Blok BM, de Greef MH, ten Hacken NH, Sprenger SR, Postema K, Wempe JB. The effects of a lifestyle physical activity counseling program with feedback of a pedometer during pulmonary rehabilitation in patients with COPD: a pilot study. Patient Educ Couns. 2006;61(1):48-55.

89. de Godoy DV, de Godoy RF. A randomized controlled trial of the effect of psychotherapy on anxiety and depression in chronic obstructive pulmonary disease. Arch Phys Med Rehabil. 2003;84(8):1154-1157.

90. Donesky-Cuenco D, Nguyen HQ, Paul S, Carrieri-Kohlman V. Yoga therapy decreases dyspnea-related distress and improves functional performance in people with chronic obstructive pulmonary disease: a pilot study. J Altern Complement Med. 2009;15(3):225-234.

91. Effing T, Zielhuis G, Kerstjens H, van der Valk P, van der Palen J. Community based physiotherapeutic exercise in COPD self-management: a randomised controlled trial. Respir Med. 2011;105(3):418-426.

92. Elçi A, Börekçi S, Ovayolu N, Elbek O. The efficacy and applicability of a pulmonary rehabilitation programme for patients with COPD in a secondary-care community hospital. Respirology. 2008;13(5):703-707.

93. Emery CF, Schein RL, Hauck ER, MacIntyre NR. Psychological and cognitive outcomes of a randomized trial of exercise among patients with chronic obstructive pulmonary disease. Health psychology: official journal of the Division of Health Psychol. 1998;17(3):232-240.

94. Gift AG, Moore T, Soeken K. Relaxation to reduce dyspnea and anxiety in COPD patients. Nurs Res. 1992;41(4):242-246.

95. Griffiths TL. Results at 1 year of outpatient multidisciplinary pulmonary rehabilitation: a randomised controlled trial. Lancet. 2000;355(9211):1280.

96. Guell R, Resqueti V, Sangenis M, et al. Impact of pulmonary rehabilitation on psychosocial morbidity in patients with severe COPD. Chest. 2006;129(4):899-904.
97. Hospes G, Bossenbroek L, ten Hacken NH, van Hengel P, de Greef MH. Enhancement of daily physical activity increases physical fitness of outclinic COPD patients: results of an exercise counseling program. Patient Educ Couns. 2009;75(2):274-278.

98. Hynninen MJ, Bjerke N, Pallesen S, Bakke PS, Nordhus IH. A randomized controlled trial of cognitive behavioral therapy for anxiety and depression in COPD. Respir Med. 2010;104(7):986-994.

99. Kapella MC, Herdegen JJ, Perlis ML, et al. Cognitive behavioral therapy for insomnia comorbid with COPD is feasible with preliminary evidence of positive sleep and fatigue effects. Int J Chron Obstruct Pulmon Dis. 2011;6:625-635.

100. Kayahan B, Karapolat H, Atýntoprak E, Atasever A, Oztürk O. Psychological outcomes of an outpatient pulmonary rehabilitation program in patients with chronic obstructive pulmonary disease. Respir Med. 2006;100(6):1050-1057.

101. Kunik ME, Braun U, Stanley MA, et al. One session cognitive behavioural therapy for elderly patients with chronic obstructive pulmonary disease. Psychol Med. 2001;31(4):717-723.

102. Kunik ME, Veazey C, Cully JA, et al. COPD education and cognitive behavioral therapy group treatment for clinically significant symptoms of depression and anxiety in COPD patients: a randomized controlled trial. Psychol Med. 2008;38(3):385-396.

103. Lamers F, Jonkers CC, Bosma H, Chavannes NH, Knottnerus JA, van Eijk JT. Improving quality of life in depressed COPD patients: effectiveness of a minimal psychological intervention. COPD. 2010;7(5):315-322.

104. Livermore N, Sharpe L, McKenzie D. Prevention of panic attacks and panic disorder in COPD. Eur Respir J. 2010;35(3):557-563.

105. Lolak S, Connors GL, Sheridan MJ, Wise TN. Effects of progressive muscle relaxation training on anxiety and depression in patients enrolled in an outpatient pulmonary rehabilitation program. Psychother Psychosom. 2008;77(2):119-125.

106. Lord VM, Cave P, Hume VJ, et al. Singing teaching as a therapy for chronic respiratory disease - a randomised controlled trial and qualitative evaluation. BMC Pulm Med. 2010;10:41.

107. McGeoch GRB, Willsman KJ, Dowson CA, et al. Self-management plans in the primary care of patients with chronic obstructive pulmonary disease. Respirology. 2006;11(5):611-618.

108. Ozdemir EP, Solak O, Fidan F, et al. The effect of water-based pulmonary rehabilitation on anxiety and quality of life in chronic pulmonary obstructive disease patients. Turk Klin Tip Bilim. Jun 2010; 30(3):880-887.

109. Paz-Diaz H, Montes de Oca M, López JM, Celli BR. Pulmonary rehabilitation improves depression, anxiety, dyspnea and health status in patients with COPD. Am J Phys Med Rehabil. 2007;86(1):30-36.

110. Sassi-Dambron DE, Eakin EG, Ries AL, Kaplan RM. Treatment of dyspnea in COPD. A controlled clinical trial of dyspnea management strategies. Chest. 1995;107(3):724-729.

111. Spencer LM, Alison JA, McKeough ZJ. Maintaining benefits following pulmonary rehabilitation: a randomised controlled trial. Eur Respir J. 2010;35(3):571-577.

112. Taylor SJC, Sohanpal R, Bremner SA, Devine A, Eldridge S, Griffiths CJ. Pilot randomised controlled trial of a 7-week diseasespecific self-management programme for patients with COPD: BELLA (Better Living with Long term Airways disease study). Thorax. 2009;64:A95-A97.

113. Yeh GY, Roberts DH, Wayne PM, Davis RB, Quilty MT, Phillips RS. Tai chi exercise for patients with chronic obstructive pulmonary disease: a pilot study. Respir Care. 2010;55(11):1475-1482.

114. Ries AL, Kaplan RM, Limberg TM, Prewitt LM. Effects of pulmonary rehabilitation on physiologic and psychosocial outcomes in patients with chronic obstructive pulmonary disease. Ann Int Med. 1995;122(11):823-832. 
International Journal of COPD

Dovepress

\section{Publish your work in this journal}

The International Journal of COPD is an international, peer-reviewed journal of therapeutics and pharmacology focusing on concise rapid reporting of clinical studies and reviews in COPD. Special focus is given to the pathophysiological processes underlying the disease, intervention programs, patient focused education, and self management protocols.

This journal is indexed on PubMed Central, MedLine and CAS. The manuscript management system is completely online and includes a very quick and fair peer-review system, which is all easy to use. Visit http://www.dovepress.com/testimonials.php to read real quotes from published authors.

Submit your manuscript here: http://www.dovepress.com/international-journal-of-copd-journal 